Supplementary Information for:

Structural Invariance in Silver(I) Coordination Networks formed using Flexible FourArmed Thiopyridine Ligands

David B. Cordes and Lyall R. Hanton*

\title{
Topological Analysis
}

The topological analysis of all six complexes was conducted using the program OLEX. ${ }^{1} \quad$ All structures displayed two topologically different nodes, one sixconnected and based on the centroid of the benzene ring of the ligand, the other threeconnected and based on Ag.

The three-dimensional frameworks of 1-3 were found to be topologically equivalent. The six-connected nodes were found to have the short topological term of $6^{12} .8^{3}$ while the three-connected nodes had short topological terms of $6^{3}$. This network topology is also referred to as the pyrite net. The long topological terms for these nodes are $6.6 \cdot 6 \cdot 6_{2} \cdot 6 \cdot 6_{2} \cdot 6 \cdot 6_{2} \cdot 6 \cdot 6_{2} \cdot 6_{2} \cdot 6_{2}$ and $6_{3} \cdot 6_{3} \cdot 6_{3}$, respectively.

The three-dimensional frameworks of both $\mathbf{4}$ and $\mathbf{5}$ were found to be topologically equivalent. The six-connected nodes were found to have the short topological term of $4^{2} .6^{10} .8^{3}$ while the three-connected nodes had short topological terms of $4.6^{2}$. This network topology is also referred to as the rutile net. The long topological terms for these nodes are 4.4.6.6.6.6.6.6.6.6.6. $6.6_{2}$ and $6.6_{2} \cdot 6_{2}$, respectively.

The three-dimensional framework of $\mathbf{6}$ had six-connected nodes with short topological terms of $4^{4} \cdot 6^{2} \cdot 8^{7} \cdot 10^{2}$ and three-connected nodes with short topological terms of $4^{2} .6$. The long topological terms of these nodes are 4.4.4.4.8.8 $2.8 .8_{2} \cdot 8_{2} .8_{3} .8_{3} .8_{4}$ and $4.4 .8_{6}$, respectively.

\section{References}

(1) OLEX, Dolomanov, O. V.; Blake, A. J.; Champness, N. R.; Schröder, M. J. Appl. Cryst. 2003, 36, 1283. 